\title{
PERFIL SOCIODEMOGRÁFICO E ESTADO DE SAÚDE AUTO-REFERIDO ENTRE IDOSAS DE UMA LOCALIDADE DE BAIXA RENDA
}

\author{
Socio-demographic profile and self-reported health status of \\ elderly women in a low-income community \\ Perfil sociodemográfico y estado de salud auto-referido \\ entre ancianas de una comunidad de escasos recursos.
}

\section{RESUMO}

Os objetivos foram elaborar diagnóstico sociodemográfico e identificar a auto-percepção quanto ao estado de saúde de mulheres idosas de uma localidade de baixa renda do município do Rio de Janeiro, Brasil. Trata-se de estudo descritivo, de corte seccional, desenvolvido em três momentos: grupos focais exploratórios; Censo da População Idosa e inquérito domiciliar com as idosas identificadas no Censo. Os sujeitos foram 369 idosas, correspondendo a 83,5\% da população elegível. As mulheres corresponderam a $62,8 \%$ do total de idosos da comunidade, apresentando uma condição social nada favorável (49,3\% são analfabetas, 44,7\% estão viúvas, 62,3\% tem renda mensal de até um salário mínimo e 22,0\% declararam não ter renda). Apesar de $62,0 \%$ afirmarem que têm uma boa saúde, $80,5 \%$ referiram apresentar algum problema de saúde. Pela sua própria condição (idosa, mulher e pobre), o grupo já se encontra potencialmente numa situação de risco e de desvantagem social em relação a outros grupos.

Palavras-chave: Saúde do Idoso. Saúde da Mulher. Envelhecimento. Estudos Transversais.

\begin{abstract}
The main objective of the present study is to develop a sociodemographic diagnosis to identify the self-perception of the state of health of elderly women in a community of low income in a county of the city of Rio de Janeiro, Brazil. It is a descriptive study, developed in three stages: exploratory focus groups; a Census of the elderly population and a survey with the elderly women identified in the Census. The subjects were 369 elderly women, number that corresponds to $83.5 \%$ of the eligible population. Women accounted for $62.8 \%$ of the total of the elderly in thecommunity, presenting a disfavorable social condition (49.3\% are illiterate, $44.7 \%$ are widows, $62.3 \%$ have a monthly income of up to a minimum wage and $22,0 \%$ reported not having an income at all). Even though $62.0 \%$ say they have good health, $80.5 \%$ say they have some type of health problem. Due to their condition (elderly, women and poor), the group is already potentially in a situation of risk and social disadvantage compared to other groups.
\end{abstract}

Keywords: Health of the Elderly. Women's Health. Aging. CrossSectional Studies.

\section{Resumen}

El objetivo fue elaborar un perfil sociodemográfico e identificar la auto-percepción en relación al estado de salud de mujeres ancianas que viven en una comunidad de escasos recursos de un municipio de Rio de Janeiro, Brasil. Se trata de un estudo de tipo descriptivo, de corte seccional, desarrollado en tres momentos: grupos focales exploratórios; Censo de la población anciana y encuestas en los domicilios con las ancianas identificadas en el Censo. Los sujetos del estudio fueron 369 ancianas, lo que corresponde a 83,5\% de la población elegible . Las mujeres correspondieron a 62,8\% del total de ancianos de la comunidad y presentan una condición social nada favorable $(49,3 \%$ son analfabetas, $44,7 \%$ son viudas, $62,3 \%$ tienen una renta mensual de hasta un salario mínimo y $22,0 \%$ declararon no tener ningún ingreso ). A pesar de que $62,0 \%$ de las ancianas aseguren contar con una buena salud, $80,5 \%$ afirmaron que tienen algún problema de salud. Por su condición propia (anciana, mujer y pobre), el grupo ya se encuentra potencialmente en una situación de riesgo y desventaja social en lo referente a otros grupos.

Palabras-claves: Salud del Anciano. Salud de la Mujer. Envejecimiento Estudios Transversales. 


\section{INTRODUÇÃO}

0 Brasil, até pouco tempo, era considerado um país de jovens, mas este perfil vem se modificando ao longo das últimas décadas. 0 percentual de pessoas com 60 anos ou mais de idade representava $4 \%$ da população total do país em 1940 , passando a 7,3\% no Censo Demográfico de 1991 e 8,6\% no Censo $2000^{1,2}$. Apesar de a proporção de idosos ${ }^{b}$ na população geral continuar sendo relativamente pequena, quando comparada a alguns países desenvolvidos, o número absoluto de idosos está aumentando com rapidez (10.722.705 em 1991 para 14.536.029 em 2001) e já se encontra entre os cinco maiores do mundo, número superior ao de países como França, Itália e Reino Unido!.

No entanto, este aumento não tem sido uniforme em ambos os sexos. Na América Latina e Caribe, como vem ocorrendo em outras partes do mundo, as previsões apontam que as mulheres continuarão tendo uma vantagem na esperança de vida em relação aos homens ${ }^{3}$. Este fenômeno é o que alguns autores estão chamando de "feminização da terceira idade" As estatísticas apontam que, em média, as mulheres brasileiras vivem oito anos mais que os homens ${ }^{1}$.

No Brasil, as propostas para a assistência à mulher se concretizaram com a elaboração do Programa de Saúde Materno-Infantil (PSMI) em 19735. 0 PSMI foi implantado no conjunto das políticas de extensão de cobertura durante 0 período do regime militar, e suas ações enfatizavam a prevenção e os cuidados básicos como: acompanhamento do pré-natal, controle dos partos domiciliares, controle do puerpério, promoção da saúde da criança, dentre outros ${ }^{6}$. Em 1984, o PSMI foi desdobrado no Programa de Atenção Integral à Saúde da Mulher (PAISM) e no Programa de Atenção Integral à Saúde da Criança (PAISC). A implantação do PAISM ocorreu em um momento de abertura política no qual crescia um sentimento de democratização devido à organização de movimentos sociais. A saúde da mulher passou a ser tema de interesse não apenas nos espaços acadêmicos, mas também nos movimentos sociais organizados ${ }^{7}$.

0 Ministério da Saúde, ao formular o PAISM, o situa no contexto da política de expansão e consolidação dos serviços básicos de saúde, fazendo parte de uma proposta global de assistência primária à saúde da população, que tem como referência a questão da integralidade da assistência ${ }^{8}$. Esta procura da integralidade diferencia a proposta do PAISM das anteriores, que enfocavam a mulher apenas quanto à sua saúde reprodutiva. No entanto, uma análise mais detalhada dos objetivos programáticos do PAISM evidencia que a última etapa do ciclo da vida continuou sendo pouco considerada, com exceção das atividades de prevenção e controle do câncer do colo útero e de mama, e a identificação e controle de outras patologias de maior prevalência no grupo.

Embora tenha havido uma mudança no discurso estatal com relação à conceituação de assistência à mulher (de mãe para mulher), enquanto programa específico, na operacionalização deste, a assistência prestada continuou tendo um enfoque biológico e curativo, e a oferta de serviços é limitada na esfera pública?.

Com vistas a proporcionar informações que possam subsidiar o estabelecimento de políticas e programas voltados para promoção da saúde e qualidade de vida das mulheres idosas de localidades de baixa renda, o presente estudo teve como objetivos elaborar diagnóstico sociodemográfico e captar a autopercepção quanto ao estado de saúde entre idosas de baixa renda.

\section{METODOLOGIA}

Trata-se de um estudo descritivo, de corte seccional, desenvolvido em duas fases: um censo para identificação do universo de idosos e um inquérito domiciliar com as mulheres idosas identificadas no censo (Figura 1).

A área estudada foi a Nova Holanda, uma das 16 comunidades do Bairro Maré, XXXª Região Administrativa, situada na região da Leopoldina do município do Rio de Janeiro, Brasil. Tal escolha se deu em função não somente da carência de estudos com população idosa de áreas de baixa renda, mas, principalmente, pela possibilidade de aplicação e utilização dos resultados na solução dos problemas enfrentados pelos idosos das comunidades que compõem o Bairro Maré.

A primeira etapa compreendeu a realização do Censo da População Idosa da Nova Holanda e teve como objetivo localizar e identificar a população idosa residente na comunidade. A coleta foi realizada em 1999, por quatro moradores do Bairro Maré, que foram selecionados, treinados e supervisionados pela pesquisadora. Todos os imóveis da comunidade Nova Holanda foram visitados, perfazendo um total de 3.597. Destes, 3.259 eram residenciais (Figura 1).

Com os dados do Censo, foi criado um banco de dados, utilizando-se o software Epi-Info (versão 6.04). As informações, depois de processadas, foram apresentadas em tabelas, com freqüências simples e percentual. Para a localização da população elegível para o estudo, isto é, todas as mulheres idosas residentes na comunidade com 60 anos ou mais de idade até dezembro/1999, foi criado um segundo banco somente com os dados dos idosos identificados (Figura 1).

A segunda etapa foi realizada em 2000 e dirigida às 442 idosas localizadas. Estas foram visitadas em seus domicílios por entrevistadores treinados para aplicação de um questionário. Excetuando-se os casos de recusas e perdas, o percentual de idosas entrevistadas foi de 83,5\% (369), da população elegível (Figura 1).

Para subsidiar a elaboração do questionário, foram realizadas discussões em grupo (grupos focais) com o grupo de convivência do Centro Municipal de Atendimento Social Integrado de Nova Holanda (CEMASI), do qual participavam 120 idosos. 
A elaboração do questionário foi precedida, também, da realização de uma revisão bibliográfica sobre estudos desenvolvidos com idosos do Brasil e do exterior. Foram pesquisados questionários testados em outros estudos epidemiológicos, os quais demonstraram bons resultados de validade e confiabilidade, como o Brazil Old Age Schedule (BOAS). 0 BOAS é um questionário de avaliação funcional multidimensional, utilizado em um estudo epidemiológico, realizado no ano de 1989 , sob a coordenação de Veras ${ }^{10}$, abrangendo uma amostra de idosos de três bairros do município do Rio de Janeiro.

0 questionário construído tem 73 perguntas fechadas e abertas, contendo as variáveis: informações sociodemográficas (idade, situação conjugal, escolaridade, renda e tamanho da família), estado de saúde auto-referido e morbidade referida. Este questionário foi pré-testado e aprimorado. Com o objetivo de testar a logística do estudo como um todo, foi realizado um estudo-piloto, concomitante ao processo de treinamento dos entrevistadores.

A pesquisa foi aprovada pelo Comitê de Ética em Pesquisa do Instituto Fernandes Figueira/FIOCRUZ. Em atendimento à Resolução 196/96 do Conselho Nacional de Saúde, foi anexado ao questionário um Termo de Consentimento Livre e Esclarecido para que as idosas pudessem autorizar sua participação voluntária.

Após a aplicação dos questionários, estes foram revisados e codificados. A seguir, procederam a digitação dupla independente utilizando-se o software Epi-Info 6 e correção das inconsistências identificadas. A análise estatística foi realizada através do software SPSS for Windows (Statistical Package for the Social Sciences), versão 9.0. Diferenças entre proporções foram testadas através do teste Qui-Quadrado $\left(\chi^{2}\right)$, com nível de significância de 0,05.

\section{Figura 1}

Diagrama de Constituição da População do Estudo

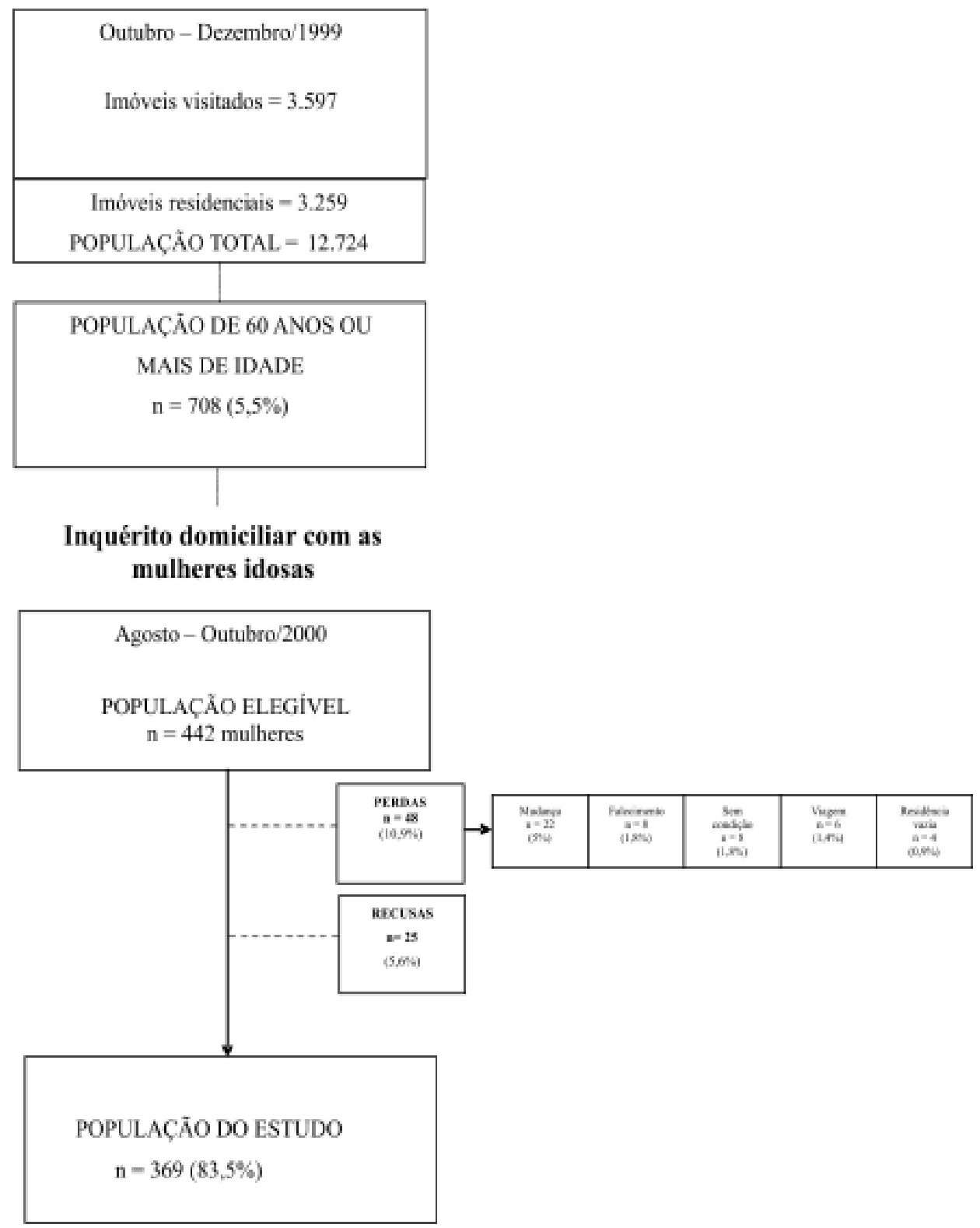




\section{RESULTADOS E DISCUSSÃO}

Nas 3.259 unidades domiciliares existentes na Nova Holanda, foram encontrados 12.724 habitantes (Figura 1), sendo $34,2 \%$ menores de 15 anos, $60,3 \%$ de 15 a 59 anos e $5,5 \%$ de 60 anos ou mais. 0 número de pessoas por domicílio variou de 1 a 28, com uma média de 3,9 moradores por domicilio $(\mathrm{DP}=2,26)$. A densidade domiciliar é um dos indicadores da qualidade de vida da população. No Brasil, em 2006, o número médio de pessoas por domićílio ficou em 3,4 ${ }^{11}$.

Foram identificados 708 idosos, correspondendo a um percentual de 5,5\% em relação à população total da comunidade, o que está abaixo da proporção nacional $(8,6 \%$ em 2000) ${ }^{1}$ e do estado do Rio de Janeiro (14,1\% em 2006) ${ }^{11}$.

Do total de unidades domiciliares, 593 tinham idosos, equivalente a $18,2 \%$ das mesmas. 0 número de idosos por domicílio variou de 1 a 3 , sendo que a predominância foi de domicílios com apenas um idoso ( $81,3 \%$ dos mesmos). Em 117 destes domicílios residiam apenas idosos, sendo que na maioria $(76,9 \%)$ residia apenas um idoso, o que significa que na comunidade há 90 idosos vivendo sozinhos, constituindo-se um grupo de maior risco e que, portanto, necessita de mais atenção e cuidado.

Quanto à distribuição por grupos de idade, observou-se que esta variou de 60 a 91 anos, sendo que 65,9\% estão no grupo de 60 a 69 anos, seguidos de $26,0 \%$ no de 70 a 79 anos e $8,0 \%$ com idade de 80 anos ou mais. Esta concentração de idosos no grupo de 60 a 69 anos é uma característica do recente processo de envelhecimento brasileiro, diferenciando-se do que vem ocorrendo nos países desenvolvidos, onde a concentração é maior no grupo de 80 anos ou mais.

As mulheres representam a maioria $(62,4 \%=442)$ dos idosos entrevistados. Este é um dado importante, pois os problemas dessas mulheres representam, conseqüentemente, os problemas dos idosos da comunidade. Os diferenciais por gênero já foram observados na Pesquisa Nacional por Amostra de Domicílios (PNAD) $2006^{11} \mathrm{e}$ em outros estudos realizados com populações idosas, tanto no Brasil2, 10, ${ }^{12}$ quanto em outros países $^{3,13}$.

As 442 idosas identificadas no Censo, ao serem revisitadas para aplicação do questionário, apresentaram um percentual de 5,6\% (25) de recusa. Além disso, mais 10,9\% (48) não foram entrevistadas por diversos motivos (Figura 1), resultando em um total de 369 , correspondendo a $83,5 \%$ das idosas da comunidade.

\section{Perfil sociodemográfico das mulheres idosas}

As características sociodemográficas das entrevistadas são apresentadas na Tabela 1. A idade variou de 60 a 90 anos, com uma média de 69 anos ( $\mathrm{DP}=6,73$ ). A concentração no grupo etário de 60 a 69 anos segue a tendência já encontrada entre todos os idosos da Nova Holanda, que, como já foi dito, é uma característica do processo de envelhecimento brasileiro.
Quanto à situação conjugal, cabe destacar a proporção de idosas viúvas que foi de 44,7\%. 0 tempo de viuvez variou de 3 meses a 42 anos, com média de 17 anos (DP=12,29). Esse resultado coincide com os dados do Censo Demográfico de 2000, que mostrou que $40,8 \%$ das idosas brasileiras se encontravam viúvas, o que, segundo Camarano et al. ${ }^{2}$, se deve à maior longevidade da mulher e ao recasamento mais comumente observado entre os homens. Apesar de uma pequena variação entre os países, tal situação é encontrada na América Latina, de um modo geral. Como conseqüência, essas mulheres acabam assumindo o papel de chefe de família e todos os encargos financeiros dele decorrentes ${ }^{3}$.

0 percentual de idosas casadas ou em união consensual foi de 28,7\%. 0 tempo de casada variou de 2 a 62 anos, com média de 35 anos $(\mathrm{DP}=13,4)$. Já quando são somados os percentuais de idosas viúvas com as solteiras $(19,0 \%)$ e as divorciadas/separadas (7,6\%), chega-se a um total de $71,3 \%$ de mulheres sem cônjuge. Para alguns pesquisadores, este é um fato preocupante na medida em que "a presença do cônjuge pode ser muito importante para a segurança e estabilidade financeira e afetiva dos idosos"14:64. No entanto, o que se observa atualmente é que as mulheres quando ficam viúvas ou se separam têm uma maior capacidade de adaptação a esta nova condição, tornando-se independentes e assumindo as responsabilidades financeiras decorrentes da vida sem cônjuge.

0 percentual de idosas analfabetas (incluídas as que declararam não saber ler e escrever e as que só sabem assinar o próprio nome) foi de $49,3 \%$. Proporcionalmente os homens idosos continuam mais alfabetizados do que as mulheres. No Brasil, em 2000, o percentual de analfabetos entre idosos era de $35,2 \%$, sendo $32,2 \%$ entre os homens e $37,4 \%$ entre as mulheres ${ }^{1}$. Marin, Ricci, Cecilio, Druzian e Rodrigues ${ }^{15}$ em estudo realizado com idosas residentes na área de abrangência de uma Unidade de Saúde da Família do interior de São Paulo também encontraram um alto percentual de idosas analfabetas $(59,4 \%)$.

0 nível de escolaridade das idosas de Nova Holanda é muito baixo. Das $50,7 \%$ que declararam saber ler e escrever, a maioria não completou sequer a primeira fase do ensino fundamental. Essa baixa escolaridade é evidenciada também pelos seguintes números: das 19 que conseguiram chegar a $2^{\mathrm{a}}$ fase do ensino fundamental, apenas 3 concluíram a referida fase; das 2 que conseguiram chegar ao ensino médio, apenas 1 concluiu o curso e nenhuma teve acesso ao ensino superior. Entre os idosos brasileiros o número médio de anos de estudo foi e 3,8 (4,0 entre as mulheres e 3,7 entre os homens) $)^{11}$.

Há que ser considerado o fato de que essas idosas nasceram e cresceram num período em que a dificuldade de acesso à educação era muito grande, principalmente para as mulheres, pois a prioridade era para os homens. Esses resultados não são uma exclusividade dos idosos, muito menos da população de Nova Holanda, mas são o reflexo dos baixos níveis educacionais vigentes no Brasil até recentemente. 
Segundo Sennot-Miller:117, o grau de escolaridade mantém íntima relação com melhores perspectivas econômicas, uma família menor e saudável, e uma melhor nutrição e saúde em geral.

Quanto à cor ou raça das entrevistadas, cabe destacar o alto percentual de idosas que se autodeclararam pardas $(48,0 \%)$. 0 restante se autodeclarou preta $(27,9 \%)$, branca $(23,8 \%)$ e amarela (0,3\%). Já a PNAD de 2006 mostrou que no Brasil a proporção de idosos é a seguinte: $57,2 \%$ de brancos e $41,6 \%$ de pardos e pretos ${ }^{16}$.
A grande maioria $(95,1 \%)$ afirmou professar alguma religião. Destas, 58,7\% declararam ser católicas, 39,9\% protestantese $1,4 \%$ espíritas.

$\mathrm{Na}$ comunidade não foi encontrada nenhuma idosa de origem estrangeira. Quanto à naturalidade, a maioria das idosas $(60,7 \%)$ é procedente de estados da própria região sudeste, sendo que mais da metade destas vem de municípios do interior do Rio de Janeiro. Outros $37,7 \%$ são provenientes da região nordeste. Segundo Chaimowicz ${ }^{14}$, a situação financeira atual dos idosos que emigraram ainda jovens para os grandes centros urbanos é tão ruim quanto aquela que motivou a migração.

\begin{tabular}{|c|c|c|}
\hline \multicolumn{3}{|c|}{$\begin{array}{l}\text { Características sociodemográficas das mulheres idosas residentes na comunidade Nova Holanda, Bairro } \\
\text { Maré, Município do Rio de Janeiro, } 2000(n=369)\end{array}$} \\
\hline & $n$ & $\%$ \\
\hline \multicolumn{3}{|l|}{ 1. Subgrupos etários } \\
\hline $60-69$ anos & 221 & 59,9 \\
\hline $70-79$ anos & 110 & 29,8 \\
\hline $80+$ anos & 38 & 10,3 \\
\hline \multicolumn{3}{|l|}{ 2. Situaçāo conjugal } \\
\hline Viúva & 165 & 44,7 \\
\hline Casada/uniāo consensual & 106 & 28,7 \\
\hline Solteira & 70 & 19,0 \\
\hline Divorciada/separada & 28 & 7,6 \\
\hline \multicolumn{3}{|l|}{$\begin{array}{l}\text { 3. Nivel educacional } \\
\text { Alfabetizaçāo }\end{array}$} \\
\hline $\begin{array}{l}\text { Alfabetizaçāo } \\
\text { Sabe ler e escrever }\end{array}$ & 187 & 50,7 \\
\hline Não sabe ler e escrever & 108 & 29,3 \\
\hline Só escreve o nome & 74 & 20,0 \\
\hline \multicolumn{3}{|l|}{ Grau de Escolaridade } \\
\hline Nunca freqüentou a escola & 139 & 37,7 \\
\hline Ensino fundamental $-1^{\circ}$ fase (incompleto) & 193 & 52,3 \\
\hline Ensino fundamental - $1^{\circ}$ fase (completo) & 16 & 4,3 \\
\hline Ensino fundamental - $2^{\circ}$ fase (incompleto) & 16 & 4,3 \\
\hline Ensino fundamental $-2^{\circ}$ fase (completo) & 3 & 0,8 \\
\hline Ensino médio incompleto & 1 & 0,3 \\
\hline Ensino médio completo & 1 & 0,3 \\
\hline \multicolumn{3}{|l|}{ 4. Raça/cor } \\
\hline Parda & 177 & 48,0 \\
\hline Preta & 103 & 27,9 \\
\hline Branca & 88 & 23,8 \\
\hline Amarela & 1 & 0,3 \\
\hline \multicolumn{3}{|l|}{ 5. Religião } \\
\hline Tem religiẫo & 351 & 95,1 \\
\hline Nāo tem religiāo & 18 & 4,9 \\
\hline \multicolumn{3}{|l|}{ 6. Naturalidade } \\
\hline SUDESTE & 224 & 60,7 \\
\hline Rio de Janeiro & 127 & \\
\hline Minas Gerais & 78 & \\
\hline Espírito Santo & 16 & \\
\hline \multicolumn{3}{|l|}{ Sāo Paulo } \\
\hline NORDESTE & 139 & 37,7 \\
\hline Paraiba & 58 & \\
\hline Pernambuco & 23 & \\
\hline Bahia & 23 & \\
\hline Rio Grande do Norte & 13 & \\
\hline Alagoas & 8 & \\
\hline Ceará & 6 & \\
\hline Sergipe & 5 & \\
\hline Maranhāo & 2 & \\
\hline Piauí & 1 & \\
\hline NORTE & 2 & 0,5 \\
\hline Amazonas & 1 & \\
\hline Pará & 1 & \\
\hline SUL & 2 & 0,5 \\
\hline Rio Grande do Sul & 2 & \\
\hline CENTRO-OESTE & 1 & 0,3 \\
\hline $\begin{array}{l}\text { Mato Grosso do Sul } \\
\text { Não sabe }\end{array}$ & 1 & 0,3 \\
\hline
\end{tabular}


Quanto à renda, foram coletadas informaç̃̃es sobre salários e outras fontes de renda das idosas e a renda mensal familiar. A renda mensal das entrevistadas foi agrupada em frações do salário mínimo vigente em dezembro/2000, que era de $\mathrm{R} \$$ 151,00 , equivalente a 76,28 dólares americanos pela cotação de 01/12/00, da seguinte forma: de 0,01 a 1; de 1,01 a 2; e de 2,01 a 4. Na Nova Holanda, o rendimento mensal das idosas variou de 0 a 4 salários mínimos, sendo a média de 0,96 $(\mathrm{DP}=0,72)$. Destas, $22 \%$ não têm renda e $62,3 \%$ têm renda de até 1 salário mínimo (Tabela 2).

Entre as idosas que declararam ter renda, $84,7 \%$ têm como principal fonte de renda as pensões ou aposentadorias (Tabela 2). Tal resultado evidencia a importância desses dois benefícios previdenciários para a sobrevivência das idosas. Camarano, Kanso e Melo $^{2}$ mostraram que a importância das aposentadorias e pensões na renda das pessoas idosas tem crescido ao longo do período 1980 a 2000, tanto para homens quanto para mulheres.

A renda mensal familiar das entrevistadas foi, também, agrupada em frações do salário mínimo vigente em dezembro/ 2000, da seguinte maneira: de 0,01 a 1; de 1,01 a 2; de 2,01 a 3; e de 3;01 a 7. Constatou-se que esta variou de 0 a 7 salários mínimos, sendo a média de 1,86 (DP=1,14). A maioria das famílias das idosas tem rendimentos de até 2 salários mínimos (Tabela 2). Já a renda familiar per capita variou de 0 a 4 salários mínimos, sendo que a média encontrada foi de $0,74$ do salário mínimo ( $\mathrm{DP}=0,56)$.

A desigualdade de renda é uma característica da população brasileira e não seria diferente entre os idosos, pelo contrário, a situação desse grupo é ainda pior. No Brasil, embora 34,3\% de idosos tenham renda mensal familiar per capita de até um salário mínimo, 12,4\% viviam com até meio salário mínimo, 0 que os caracteriza como absolutamente pobres ${ }^{16}$.

Quando analisada a contribuição dos rendimentos das idosas na renda familiar, constata-se que elas são responsáveis por um percentual importante na renda das suas famílias, correspondendo a uma média de 58,8\% ( $D P=39,7$ ). Considerando que a principal fonte de seus rendimentos são as pensões e aposentadorias, pode-se inferir que os reflexos (positivos ou negativos) do sistema previdenciário brasileiro atingem não somente os rendimentos de indivíduos, mas de famílias inteiras. Resultados semelhantes foram encontrados no Censo de 2000, o qual evidenciou que essa contribuição foi de $58,5 \%$ nas famílias que contêm idosos ${ }^{2}$.

\section{Tabela 2}

Renda mensal e respectiva fonte das entrevistadas - Nova Holanda, Bairro Maré, Município do Rio de Janeiro, 2000

\begin{tabular}{lrr}
\hline Renda mensal em salários mínimos $(\mathrm{n}=369)$ & $\mathrm{n}$ & $\%$ \\
\hline Sem renda & 81 & 22,0 \\
De 0,01 a 1 & 230 & 62,3 \\
De 1,01 a 2 & 42 & 11,4 \\
De 2,01 a 4 & 16 & 4,3 \\
Fonte(s) de renda $(\mathrm{n}=288)$ & & \\
Pensão ou aposentadoria & 270 & 93,8 \\
Trabalho formal & 33 & 11,4 \\
Trabalho informal & 5 & 1,7 \\
Marido & 1 & 0,3 \\
Aluguel de imóvel & 1 & 0,3 \\
Outras fontes & 4 & 1,4 \\
Renda mensal familiar em salários mínimos $(\mathrm{n}=369)$ & & \\
Sem renda & 8 & 2,2 \\
De 0,01 a 1 & 140 & 38,0 \\
De 1,01 a 2 & 110 & 29,8 \\
De 2,01 a 3 & 44 & 11,9 \\
De 3,01 a 7 & 26 & 7,0 \\
Não sabe / Não respondeu & 41 & 11,1 \\
\hline
\end{tabular}

Obs.: Salário mínimo vigente em dez/2000 $=\mathrm{R} \$ 151,00$.

Obs: 26 entrevistadas relataram ter duas fontes de renda

\section{Estado de Saúde}

Para captar a percepção das idosas quanto ao seu estado de saúde, foi solicitado que as mesmas fizessem uma comparação com o estado de saúde de mulheres da mesma idade. Cerca de $62 \%$ das idosas avaliaram ter uma boa saúde, enquanto $30,1 \%$ afirmaram que atualmente sua saúde é ruim ou péssima (Tabela 3)
Comparada com outra pesquisa realizada sobre a satisfação da população com os serviços de saúde de um município do norte de Minas Gerais, dos 778 domicílios visitados, 54,0\% dos entrevistados referiram que sua saúde é muito boa ou boa, $33,9 \%$ é regular e $12,1 \%$ é ruim. No referido estudo, o percentual de pessoas na faixa etária de 60 anos ou mais foi de $16,7 \%{ }^{17}$. 
Já os resultados da pesquisa suplementar Saúde, realizada junto com a PNAD 1998 - Acesso e Utilização de Serviços de Saúde ${ }^{18}$, mostraram que $79,1 \%$ da população brasileira autoavaliaram seu estado de saúde como "muito bom ou bom" e apenas 3,6\% como "ruim ou muito ruim".

Apesar dos dois estudos citados terem sido realizados com a população em geral, os seus resultados se aproximam do encontrado entre as idosas de Nova Holanda. Um fato contraditório é que, apesar de $68,8 \%$ das mulheres terem avaliado que atualmente sua saúde é ótima ou boa, quando questionado se as entrevistadas atualmente estavam apresentando algum problema de saúde, $80,5 \%$ responderam que sim (Tabela 3).

No Censo Maré, realizado pela Universidade Federal do Rio de Janeiro (UFRJ) em 1987, do total de pessoas entrevistadas no bairro Maré, $12,42 \%$ referiram algum problema de saúde nas duas semanas anteriores à entrevista. A distribuição por sexo e faixa etária das pessoas que referiram problemas de saúde mostrou que as mulheres referiram mais problemas que os homens, resultado também encontrado na faixa etária acima de 50 anos, na qual a taxa de morbidade por 100 habitantes foi de 26,46 no sexo feminino e de 16,19 no sexo masculino ${ }^{19}$.

Quando comparada com os resultados do Censo Maré, a morbidade referida encontrada no presente estudo é bem mais alta. Dentre outros fatores, há que se considerar que o primeiro foi realizado com a população em geral de todo o bairro; e 0 segundo apenas com a população idosa de uma das comunidades.

Das que referiram problema de saúde, $53,5 \%$ apresentam um problema, 31,0\% apresentam dois e 15,5\% apresentam três problemas. Como pode ser visto na Tabela 3 , alguns dos problemas relatados não são doenças, e sim sintomas ou queixas. Os problemas mais prevalentes relatados pelas idosas foram: hipertensão arterial (47,5\%), problema de coluna $(18,8 \%)$, diabetes $(18,2 \%)$ e artrose-reumatismo $(15,1 \%)$.

Cabe destacar a diferença no perfil de morbidade ao se comparar o resultado do Censo Maré(realizado 14 anos antes) com os do presente estudo. No primeiro, os problemas mais prevalentes na população acima de 50 anos foram gripe/ resfriado, pressão alta e reumatismo, enquanto neste a presença de gripefoi referida por apenas uma das entrevistadas.

A PNAD de 1998 apontou que as pessoas apresentam mais problemas de saúde no início e no final de suas vidas. Observa ainda que no Brasil a desigualdade social em saúde é bem evidente, indicando que o número de pessoas que referem problemas de saúde diminui à medida que a renda familiar aumenta ${ }^{18}$. No entanto, na presente pesquisa não foi encontrada nenhuma associação entre renda familiar mensal e morbidade referida, provavelmente porque a renda é muito homogênea na população estudada.

Como nesse estudo foi investigada apenas a morbidade referida, não se sabe, dentre as idosas que se declararam hipertensas, quais são as que realmente foram diagnosticadas pelos serviços de saúde.

Tabela 3

\begin{tabular}{|c|c|c|}
\hline \multicolumn{3}{|c|}{$\begin{array}{l}\text { Autopercepçōo das idosas quanto ao seu atual estado de saúde e existéncia de algum } \\
\text { problema de saúde (marbidade referida) - Nova Holanda, Baimo Maré, Municipio do Rio de } \\
\text { Janeiro, } 2000\end{array}$} \\
\hline ESTADO DE SALUDE $[n=369]$ & $\mathrm{n}$ & $\%$ \\
\hline Ótima & 25 & 6,8 \\
\hline Bon & 229 & 62,0 \\
\hline Ruim & 81 & 22,0 \\
\hline Péssima & 30 & 8,1 \\
\hline Não sabe & 4 & 1,1 \\
\hline \multicolumn{3}{|l|}{ MORBIDADE REFERIDA $(n=369$ ) } \\
\hline Sim & 297 & 80,5 \\
\hline Não & 72 & 19,5 \\
\hline \multicolumn{3}{|l|}{ PROBLEMAS REFERIDOS $(n=297)$} \\
\hline Hipertensão arterial & 141 & 47,5 \\
\hline Problemo de coluno & 56 & 18,8 \\
\hline Diabetes & 54 & 18,2 \\
\hline Artrose-reumatismo & 45 & 15,1 \\
\hline Problema de visōo & 27 & 9,1 \\
\hline Problema cordioco & 26 & 8,7 \\
\hline Problema nos membros inferiores & 20 & 6,7 \\
\hline Problema digestivo & 12 & 4,0 \\
\hline Problema pulmonar & 12 & 4,0 \\
\hline Dor nos c6sos & 8 & 2,7 \\
\hline Folta de or & 5 & 1,7 \\
\hline Cegueiro & 4 & 1,3 \\
\hline Distúrbio emocional & 4 & 1,3 \\
\hline Dor de cobeca & 4 & 1,3 \\
\hline Erisipela & 4 & 1,3 \\
\hline Lobirintite & 4 & 1,3 \\
\hline Ostecporase & 4 & 1,3 \\
\hline Alergia & 3 & 1,0 \\
\hline Cansoço & 3 & 1,0 \\
\hline Problemo renal & 3 & 1,0 \\
\hline Vorizes & 2 & 0,7 \\
\hline Colesteral alto & 1 & 0,3 \\
\hline Gripe & 1 & 0,3 \\
\hline Outros & 38 & 12,8 \\
\hline
\end{tabular}




\section{(In)Satisfação com a vida}

Na sua grande maioria $(85,1 \%)$ as idosas se declararam satisfeitas com a vida. Entre as $14,9 \%$ que estão insatisfeitas, 0 principal motivo da insatisfação está relacionado a problema de saúde. Outros motivos também prevalentes foram carência de companhia e carência econômica (Tabela 4).

\section{Tabela 4}

\begin{tabular}{lrr} 
(In)Satisfação com a vida - Nova Holanda, Bairro Maré, Município do Rio de Janeiro, 2000 \\
\hline \multicolumn{1}{c}{$(n=363)^{*}$} & $n$ & $\%$ \\
\hline Satisfeita & 309 & 85,1 \\
Insatisfeita & & 14,9 \\
Motivos apontados para insatisfaçăo $(n=54)$ & 27 & 50,0 \\
Problema de saúde & 10 & 18,5 \\
Carência de companhia & 8 & 14,8 \\
Carência econômica & 2 & 3,7 \\
Carência de moradia & 2 & 3,7 \\
Carência de segurança & 5 & 9,3 \\
Outro & & 100,0 \\
\hline Total & &
\end{tabular}

* Excluídas 6 que não responderam.

\section{Autopercepção das idosas quanto ao seu atual estado de saúde e associações com variáveis selecionadas}

Para identificar possíveis associações da variável autopercepção quanto ao atual estado de saúde, esta foi correlacionada com algumas variáveis selecionadas (Tabela 5). Como as diferenças entre as categorias de respostas ótima e boa e entre ruim e péssima foram pequenas, as respostas foram fundidas da seguinte forma: ótima/boae ruim/péssima.

A análise de algumas variáveis sociodemográficas (subgrupos etários, situação conjugal, alfabetização, renda familiar mensal e participação das entrevistadas no mercado de trabalho) evidenciou associação estatisticamente significativa entre autopercepção sobre o atual estado de saúde com subgrupos etários, renda familiar mensal e participação das entrevistadas no mercado de trabalho (Tabela 5). Conforme aumenta a idade, diminui a proporção de mulheres que mencionaram ter uma saúde ótima/boa, sendo $74,7 \%$ entre as de 60 a 69 anos, $65,5 \%$ entre as de 70 a 79 anos e $52,6 \%$ entre as de 80 anos ou mais de idade $(p=0,013)$. Não foram encontradas diferenças significativas nas proporções de autopercepção sobre o atual estado de saúde segundo a situação conjugal ( $p=0,889)$. A associação com alfabetização, embora sem significância estatística ( $p=0,085)$, revelou que entre as idosas alfabetizadas é maior a proporção das que se autopercebem com uma saúde ótima/boa $(73,7 \%)$ do que as que se autopercebem com uma saúde ruim/péssima $(65,4 \%)$. Quanto maior a renda, melhor a percepção das idosas quanto ao seu estado atual de saúde $(p=0,038)$. A exceção nesse caso são as que se declararam sem renda, cujo resultado pode ter sido influenciado pelo pequeno número (oito) de respondentes nessa categoria de resposta. Entre as que trabalham, foi encontrada uma proporção maior de mulheres que referiram ter uma saúde ótima/boa $(86,5 \%)$ do que entre as que não trabalham $(67,7 \%)(p=0,018)$.

Foram também encontradas associações estatisticamente significativas com morbidade referida (se apresentam problema de saúde) e satisfação com a vida, ambas com $p=0,000$. Entre as que não têm problema de saúde, a grande maioria declarou ter saúde ótima/boa (97,2\%). As outras, apesar de referirem problema de saúde, se consideram com saúde ótima/boa (62,9\%). Dentre as satisfeitas com a vida, a maioria (76,7\%) se autopercebe uma saúde ótima/boa. 
Perfil sociodemográfico e estado de saúde auto-referido entre idosas Sousa Al, Silver LD

\section{Tabela 5}

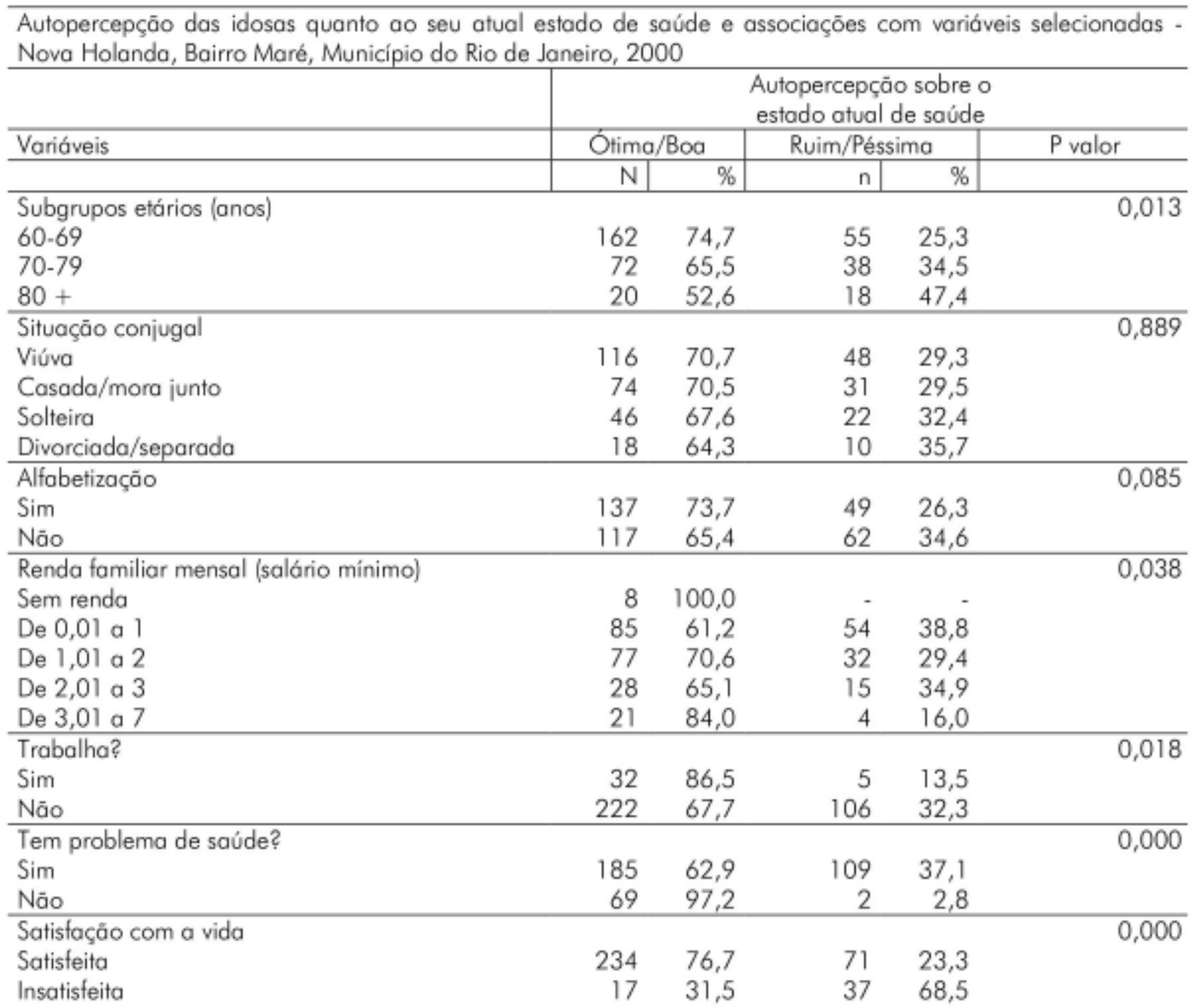




\section{CONSIDERAÇÕES FINAIS}

Este é o primeiro estudo realizado com idosas da comunidade Nova Holanda, e suas contribuições pretendem servir de subsídio para a formulação de políticas públicas voltadas para os idosos de baixa renda.

Seguiu-se aplicação rigorosa de procedimentos metodológicos durante as etapas do estudo, na busca de alcançar alta validade interna. 0 censo da população idosa mostrou-se oportuno e importante para o delineamento da pesquisa quanto ao seu limite, a identificação da população elegivel para o estudo e a construção do instrumento. Constitui um banco de dados para consultas, investigações e propostas programáticas no campo da saúde individual e coletiva. A alta adesão ao estudo possibilitou que os resultados e conclusões encontrados pudessem ser generalizados a todas as idosas da comunidade.

Estatisticamente e socialmente, o grupo estudado é de alta relevância e magnitude. Numa localidade onde grande

\section{Referências}

1. Ministério do Planejamento, Orçamento e Gestão (BR). Instituto Brasileiro de Geografia e Estatística-IBGE. Perfil dos idosos responsáveis pelos domicílios no Brasil. Rio de Janeiro (RJ): IBGE; 2002.

2.Camarano AM, Kanso S, Mello JL. Como vive o idoso brasileiro. In: Camarano AM, organizador. Os novos idosos brasileiros: muito além dos 60? Rio de Janeiro(RJ): IPEA; 2004. p. 25-73.

3.Sennott-Miller L. La mujer de edad avanzada en las Americas. Problemas y possibilidades. In: Organização Pan-Americana da Saúde.OPS. Genero, mujer y salud en las americas. Washington (USA); 1993. p. 114-123. (Publicação Científica n 541).

4.Bonita R, Howe AL. Older woman in na aging world: achieving health across the life course. Rapp Trimest Statist Sanit Mond 1996; 49: 134-41.

5.Ministério da Saúde (BR). Secretaria de Assistência Médica. Coordenação de Proteção Materno-Infantil. Programa de Saúde Materno-Intantil. Brasília (DF); 1973.

6.Gomes MASM. Aspectos da qualidade do atendimento à gestação e ao parto através da percepção das usuárias [dissertação de mestrado]. Rio de Janeiro (RJ): Instituto Fernandes Figueira/FIOCRUZ; 1995.

7.Ferreira SL. Mulher e serviços de saúde: 0 processo de trabalhos em Distritos Sanitários. Salvador (BA): Ultragraph; 1996.

8.Ministério da Saúde (BR). Assistência integral à saúde da mulher: bases de ação programática. Brasília (DF): Centro de Documentação/ MS; 1984. (Série B: Textos Básicos de Saúde, 6).

9.Tyrrell MAR, Carvalho V. Programas Nacionais de Saúde MaternoInfantil: impacto político-social e inserção da enfermagem. Rio de Janeiro (RJ): Escola de Enfermagem Anna Nery /UFRJ; 1993.

10.Veras RP. País jovem com cabelos brancos: a saúde do idoso no Brasil. $2^{\text {a }}$ ed. Rio de Janeiro (RJ): Relume Dumará/UERJ; 1994.

11.Ministério do Planejamento, Orçamento e Gestão (BR). Instituto Brasileiro de Geografia e Estatística-IBGE. Pesquisa Nacional por parte da população é constituída por jovens, para os quais não há políticas públicas específicas voltadas para seus problemas socioeconômicos, os resultados apontam que a situação das entrevistadas é ainda mais preocupante do que o encontrado nas pesquisas realizadas com os idosos brasileiros em geral. Há que se considerar que o grupo estudado pela sua própria condição (idosa, mulher e pobre) já se encontra potencialmente numa situação de risco e de desvantagem social em relação a outros grupos, fato que deveria merecer uma atenção especial por parte do poder público no sentido de diminuir a desigualdade social.

A questão social do idoso aliada à complexidade da vida em uma localidade de baixa renda exige medidas urgentes no sentido de atingir o objetivo da Política Nacional de Saúde do Idoso, que é permitir um envelhecimento saudável, preservando sua capacidade funcional, sua autonomia e mantendo o nível de qualidade de vida.

Amostra de Domicílios- PNAD. Síntese de Indicadores 2006. Rio de Janeiro(RJ): IBGE; 2007.

12.Lima e Costa MFF, Uchoa E, Guerra HL, Firmo JOA, Vidigal PG, Barreto SM. The Bambuí Health and Agening Study- BHAS: methodological approach and preliminary results of a populationbased cohort study of the elderly in Brazil. Rev Saude Publica 2000; 34( 2): 126-35.

13.Kinsella K. Dimensiones demográficas y de salud em América Latina y el Caribe. 1994. In: Organização Pan-Americana da Saúde-OPAS. La atención de los ancianos: un desafío para los años noventa. Washington (USA); 1994. p. 3-18. (Publicação Científica , 546).

14.Chaimowicz F. Os idosos brasileiros no século XXI: demografia, saúde e sociedade. Belo Horizonte (MG): Postgraduate; 1998.

15.Marin MJS, Ricci FA, Cecílio LCO, Druzian S, Rodrigues LCR. Diagnósticos de enfermagem de idosas carentes de um Programa de Saúde da Família-PSF. Esc Anna Nery Rev Enferm 2008 jun; 12(2): 278-84.

16.Ministério do Planejamento, Orçamento e Gestão (BR). Instituto Brasileiro de Geografia e Estatística- IBGE. Síntese de Indicadores Sociais. Rio de Janeiro (RJ): IBGE; 2007.

17.Sousa Al. A satisfação da população com os serviços locais de saúde - Município de Itacarambi (MG). [dissertação de mestrado]. Rio de Janeiro (RJ): Escola Nacional de Saúde Pública/FIOCRUZ; 1996.

18. Ministério do Planejamento, Orçamento e Gestão (BR). Instituto Brasileiro de Geografia e Estatística-IBGE. Pesquisa Nacional por Amostra de Domićlios-PNAD. Acesso e utilização de serviços de saúde -1998. Rio de Janeiro (RJ): IBGE; 2000.

19 - Campos CEA. A desordem da demanda: os inquéritos de morbidade, demanda e utilização sob a perspectiva do planejamento em saúde - um estudo de caso [dissertação de mestrado]. Rio de Janeiro (RJ): Escola Nacional de Saúde Pública/ FIOCRUZ; 1991 


\section{Notas}

a) Trabalho extraído da Tese de Doutorado "A visão das mulheres idosas em relação à atenção à saúde e ao apoio social em uma localidade de baixa renda do Rio de Janeiro", apresentada ao Instituto Fernandes Figueira da Fundação Oswaldo Cruz - IFF"FIOCRUZ, Rio de Janeiro (RJ), Brasil.

${ }^{b}$ Neste estudo emprega-se a categoria definida desde 1982, pela Organização das Naç̃es Unidas, e que vem sendo utilizada em países do Terceiro Mundo, de idoso como sendo toda pessoa de 60 anos ou mais de idade. 0 Brasil, na Lei 8.842/94 (Art. $2^{\circ}$ do (ap. I), adota essa mesma faixa etária. 